\title{
Separation of Aspidiotes species using morphometric analysis (Coleoptera: Curculionidae)
}

\author{
MANUEL SÁNCHEZ-RUIZ and ISABEL SANMARTÍN
}

Dpto. Biodiversidad y Biología Evolutiva Entomología, Museo Nacional de Ciencias Naturales (C.S.I.C.), c/ José Gutiérrez Abascal, 2, 28006 Madrid, Spain; e-mail: manuel_sr@mncn.csic.es; isa@mncn.csic.es

Key words. Morphometrics, taxonomy, discrimination, Aspidiotes, Curculionidae

\begin{abstract}
The efficacy of morphometric characters for separating the species of the genus Aspidiotes Schoenherr, 1847, was evaluated. Thirty characters were analyzed. Multivariate and univariate analyses of variance, and discriminant function analysis, all demonstrated that each species is morphometrically distinguishable. The lengths of rostrum, scape, onychium, pronotum, and width and length of elytra have the maximum discriminatory power. Males and females are also morphometrically distinguishable, mainly due to differences in the widths of rostrum between pterigia and at base of pronotum, and width and length of elytra. The classification functions provided by discriminant gave the correct identification of every single specimen by sex and species. Mahalanobis' distances between species were calculated and subjected to UPGMA clustering, to construct a dendrogram reflecting the morphometric relationships between species. This dendrogram did not correspond to the phylogenetic relationships depicted by a cladogram based on discrete characters (Sánchez-Ruiz \& Alonso-Zarazaga, 1994). Some hypotheses are reviewed, which might explain this discrepancy.
\end{abstract}

\section{INTRODUCTION}

The systematics of the genus Aspidiotes Schoenherr, 1847 was recently revised by Sánchez-Ruiz \& AlonsoZarazaga (1994), who found the genus was made up of six species ( $A$. anatolicus, $A$. cottyi, A. gonzalezi, $A$. larbii, $A$. thalassimus and $A$ westringii). Their cladistic analysis, based on qualitative characters of the external morphology and genitalia, suggested the existence of two subgenera within the genus, Aspidiotes and Phaenognathus. The genus Aspidiotes, as presently delimited, shows an East-West Mediterranean disjunction, well known for other taxa of plants and animals (Oosterbroek \& Arntzen, 1992).

Previously, Alonso-Zarazaga \& Sánchez-Ruiz (1990) used multivariate morphometric analysis to divide the Iberian species $A$. westringii into two allopatric species: $A$. westringii, which occurs in southeastern Spain, and a northeastern population, later described as A. gonzalezi Sanchez-Ruiz \& Alonso-Zarazaga, 1994. Differences in the external morphology and genitalia of these species were corroborated by metric characters, thus indicating the usefulness of these characters in the taxonomy of $A s$ pidiotes. In this paper we propose a new approach to the taxonomy of the genus based on an analysis of morphometric characters. This analysis reveals new taxonomic characters.

Morphometric analysis has been successfully used in other groups of insects such as aphids (Foottit, 1992; Tizado \& Nieto-Nafría, 1994), bees (Daly, 1992), planthoppers (Claridge \& Gillham, 1992), or beetles (Sanmartín \& Martín-Piera, 1999). There are, however, few studies on the systematics of Curculionidae using these techniques (Godwin et al., 1982; Horng \& Peng, 1983).
Multivariate morphometrics has proved useful not only in resolving taxonomic problems but also in coevolution studies (Houck, 1992), and even for phylogenetic inference. Sorensen \& Foottit (1992) argue that multivariate morphometric methods, in particular discriminant analysis, can be used for estimating phylogeny because, "as with cladistic methods, they evaluate partitioned variance and reflect polarity or apomorphic character states" (Sites \& Willing, 1994).

Unlike in other groups of insects, there is usually no striking (visible) sexual dimorphism in shortnosed Curculionidae. Differences between sexes in Aspidiotes involve the entire form of the insect and are thus difficult to define in terms of qualitative characters. Multivariate morphometric analysis considers simultaneously many different intercorrelated characters, thus it might help to visualize these latent, non obvious differences.

Aims of this work is: (i) evaluate morphometric characters for separating Aspidiotes species; (ii) determine the discriminatory characters for (a) every pair of species, and (b) males and females; (iii) to compare the classification of species based on this morphometric analysis with that of Sanchez-Ruiz \& Alonso-Zarazaga (1994), based on a cladistic analysis of qualitative characters.

\section{MATERIAL AND METHODS}

Table 1 shows the geographic distribution and number of specimens measured for each of the six species of genus $A s$ pidiotes. In all, 177 specimens were measured.

Thirty characters from all parts of the body of male and female adults were measured (Table 2), measurements were made with the aid of an ocular micrometer attached to a binocular microscope. Ratios or indices were not included in the analysis because of the difficulties created by using of ratios in morphometric analysis (Albretch et al., 1993). 
TABLE 1. Distribution and sample size of each species of Aspidiotes.

\begin{tabular}{llrlr}
\hline Species & Author \& date & N & Distribution & Males/Females \\
\hline A. anatolicus & (Colonnelli, 1978) & 26 & Turkey & $14 / 12$ \\
A. cottyi & (Lucas, 1858) & 51 & Morocco, Algeria & $24 / 27$ \\
A. gonzalezi & Sanchez-Ruiz \& Alonso-Zarazaga, 1994 & 20 & Northeastern Spain & $10 / 10$ \\
A. larbii & (Escalera, 1914) & 8 & Morocco & $7 / 1$ \\
A. thalassinus & (Schoenherr, 1847) & 31 & Greece, Turkey & $17 / 14$ \\
A. westringii & Schoenherr, 1847 & 41 & Southeastern Spain & $19 / 22$ \\
\hline
\end{tabular}

Statistical analyses were performed using STATISTICA 5.1 for WINDOWS (Statsoft, 1996). The basic principles of the analyses used may be found in Sneath \& Sokal (1973).

(i) We used an analysis of variance to evaluate the efficacy of morphometric characters in the separation of Aspidiotes species. First, a one-way multivariate analysis of variance (MANOVA) was performed on the six species to determine whether statistically significant differences existed between species based on the entire set of characters. One-way analyses of variance (ANOVAs) were then computed for each character to evaluate whether it contributed significantly to species differences. However, before ascribing statistical significance, we applied the sequential Bonferroni correction to each character to avoid overestimating the significance of particular characters in a large suite of attributes (Rice, 1989).

(ii) (a) The most important characters for discriminating between species in species pairs were determined by multiple discriminant function analysis (DFA). DFA demonstrated the degree of separation in multivariate space defined by the main patterns of morphological variation among species (the discriminant functions). It also showed which characters contribute more to the discrimination between species. The standardized dis-

TABLE 2. List of characters used in this study.

\begin{tabular}{ll}
\hline Character & Description \\
\hline WRP & Width of rostrum on pterigia \\
WRS & Width of rostrum between scrobes \\
LR & Length of rostrum \\
WF & Width of frons (between eyes) \\
LSC & Length of scape \\
WSC & Width of scape \\
LF1 & Length of $1^{\text {st }}$ funicular joint \\
LF2 & Length of $2^{\text {nd }}$ funicular joint \\
WF1 & Width of $1^{\text {st }}$ funicular joint \\
WF2 & Width of $2^{\text {nd }}$ funicular joint \\
LC & Length of antenal club \\
WC & Width of antenal club \\
LP & Length of pronotum (on midline) \\
WP & Width of pronotum (maximum) \\
WPB & Width of pronotum at base \\
WPA & Width of pronotum at apex \\
WE & Width of elytra (maximum) \\
LE & Length of elytra \\
LPF & Length of profemur \\
WPF & Width of profenur (maximum) \\
LPT & Length of protibia \\
WPT & Width of protibia (not including mucro) \\
LT1 & Length of $1^{\text {st }}$ protarsomere \\
LT2 & Length of $2^{\text {nd }}$ protarsomere \\
LT3 & Length of $3^{\text {rd }}$ protarsomere \\
LON & Length of onychium \\
WT1 & Width of $1^{\text {st }}$ protarsomere \\
WT2 & Width of $2^{\text {nd }}$ protarsomere \\
WT3 & Width of $3^{\text {rd protarsomere }}$ \\
WON & Width of onychium \\
\hline
\end{tabular}

criminant function coefficients (coefficients of the original variables in the discriminant functions) represent the contributions of every variable to the discriminatory power of the function; the larger the standardized coefficient, the larger the weight of the variable in the function.

In addition, DFA allows for the predictive classification of specimens. The attribution of specimens to species was checked by computing the classification functions: an individual was allocated to the species for which it had the highest classification score. The percentage of specimens properly classified is a measure of the diagnostic value of the set of characters (Foottit \& Sorensen, 1992).

(ii) (b) A similar methodology was followed to determine the existence of sexual morphometric dimorphism in the genus $\mathrm{As}$ pidiotes. First, MANOVA was used to determine if significant differences existed between males and females within the genus, regardless of species. A MANOVA test was then performed on each analysed species to test whether it presented sexual dimorphism (Planned Comparisons). An exception was made for $A$. larbii, where statistical comparison was not possible $(7$ males $/ 1$ female). Finally, discriminant analysis was used to determine the most important characters for identifying males and females within the genus.

(iii) Finally, Mahalanobis' generalized distances $\left(D^{2}\right)$ were computed between all pairs of species. The square root of Mahalanobis' distance (D) for any two species represents the length of the line between the centroids of the two species in the discriminant space (Sneath \& Sokal, 1973). Mahalanobis' distances were then subjected to the clustering method UPGMA (Unweighted Pair-Group Method Arithmetic average) to construct a dendrogram reflecting the morphometric relationships between Aspidiotes species. This dendrogram was then compared with the cladogram based on qualitative characters given by SánchezRuiz \& Alonso-Zarazaga (1994).

\section{RESULTS}

The mean value, standard deviation, and range of variation for each of the thirty characters in the six species are listed in Table 3.

(i) MANOVA showed highly significant differences (Wilks' lambda ${ }_{150.702}=0.00004 ; p<0.001$ ) between the six species based on the entire set of characters. Moreover, subsequent ANOVAs resulted in highly significant differences $(p<0.00001)$ between the species in each character, even after applying sequential Bonferroni correction. Thus, all characters contributed significantly to the separation of species.

(ii) (a) Discriminant function analysis provided five significant functions $\left(\chi^{2}=1585.089 ; p<0.000001\right)$. About $94 \%$ of the variability in the data is attributable to between-species differences when all thirty variables were considered $\left(R^{2}=0.94\right)$. The first four functions explain $97 \%$ of the total variation in the data, which is sufficient for the analysis (Table 4). 
TABLE 3. Mean value, standard deviation and range of variation of the characters measured in each species.

\begin{tabular}{|c|c|c|c|c|c|c|c|}
\hline Character & & A. anatolicus & A. cottyi & A. gonzalezi & A. larbii & A. thalassinus & A. westringii \\
\hline WRP & $\begin{array}{l}\text { Mean } \\
\text { StdDev. } \\
\text { Range }\end{array}$ & $\begin{array}{c}0.735 \\
0.046 \\
0.665-0.875\end{array}$ & $\begin{array}{c}0.836 \\
0.064 \\
0.7-0.962\end{array}$ & $\begin{array}{c}0.974 \\
0.085 \\
0.822-1.137\end{array}$ & $\begin{array}{c}0.934 \\
0.057 \\
0.822-0.997\end{array}$ & $\begin{array}{c}1.117 \\
0.093 \\
0.944-1.259\end{array}$ & $\begin{array}{c}0.818 \\
0.073 \\
0.682-0.944\end{array}$ \\
\hline WRS & $\begin{array}{l}\text { Mean } \\
\text { StdDev. } \\
\text { Range }\end{array}$ & $\begin{array}{c}0.536 \\
0.042 \\
0.472-0.647\end{array}$ & $\begin{array}{c}0.615 \\
0.053 \\
0.507-0.735\end{array}$ & $\begin{array}{c}0.683 \\
0.058 \\
0.577-0.805\end{array}$ & $\begin{array}{c}0.715 \\
0.060 \\
0.612-0.787\end{array}$ & $\begin{array}{c}0.811 \\
0.072 \\
0.680-0.962\end{array}$ & $\begin{array}{c}0.609 \\
0.056 \\
0.505-0.700\end{array}$ \\
\hline LR & $\begin{array}{l}\text { Mean } \\
\text { StdDev. } \\
\text { Range }\end{array}$ & $\begin{array}{c}1.154 \\
0.088 \\
1.01-1.361\end{array}$ & $\begin{array}{c}1.119 \\
0.082 \\
0.922-1.317\end{array}$ & $\begin{array}{c}1.363 \\
0.108 \\
1.098-1.580\end{array}$ & $\begin{array}{c}1.174 \\
0.084 \\
1.054-1.295\end{array}$ & $\begin{array}{c}1.542 \\
0.129 \\
1.273-1.712\end{array}$ & $\begin{array}{c}0.998 \\
0.094 \\
0.812-1.207\end{array}$ \\
\hline WF & $\begin{array}{l}\text { Mean } \\
\text { StdDev. } \\
\text { Range }\end{array}$ & $\begin{array}{c}0.722 \\
0.084 \\
0.595-0.997\end{array}$ & $\begin{array}{c}0.720 \\
0.084 \\
0.525-0.909\end{array}$ & $\begin{array}{c}0.851 \\
0.102 \\
0.647-1.067\end{array}$ & $\begin{array}{c}0.813 \\
0.081 \\
0.665-0.909\end{array}$ & $\begin{array}{c}1.029 \\
0.121 \\
0.840-1.278\end{array}$ & $\begin{array}{c}0.752 \\
0.091 \\
0.560-0.910\end{array}$ \\
\hline LSC & $\begin{array}{l}\text { Mean } \\
\text { StdDev. } \\
\text { Range }\end{array}$ & $\begin{array}{c}0.713 \\
0.047 \\
0.630-0.805\end{array}$ & $\begin{array}{c}0.734 \\
0.058 \\
0.595-0.909\end{array}$ & $\begin{array}{c}1.007 \\
0.080 \\
0.805-1.154\end{array}$ & $\begin{array}{c}1.052 \\
0.064 \\
0.944-1.137\end{array}$ & $\begin{array}{c}1.174 \\
0.087 \\
0.962-1.312\end{array}$ & $\begin{array}{c}0.828 \\
0.070 \\
0.700-0.962\end{array}$ \\
\hline WSC & $\begin{array}{l}\text { Mean } \\
\text { StdDev. } \\
\text { Range }\end{array}$ & $\begin{array}{c}0.155 \\
0.011 \\
0.139-0.181\end{array}$ & $\begin{array}{c}0.183 \\
0.016 \\
0.139-0.222\end{array}$ & $\begin{array}{c}0.210 \\
0.024 \\
0.167-0.250\end{array}$ & $\begin{array}{c}0.222 \\
0.017 \\
0.194-0.236\end{array}$ & $\begin{array}{c}0.206 \\
0.012 \\
0.181-0.222\end{array}$ & $\begin{array}{c}0.156 \\
0.013 \\
0.139-0.194\end{array}$ \\
\hline LFI & $\begin{array}{l}\text { Mean } \\
\text { StdDev. } \\
\text { Range }\end{array}$ & $\begin{array}{c}0.291 \\
0.021 \\
0.250-0.347\end{array}$ & $\begin{array}{c}0.315 \\
0.027 \\
0.250-0.347\end{array}$ & $\begin{array}{c}0.423 \\
0.044 \\
0.347-0.486\end{array}$ & $\begin{array}{c}0.368 \\
0.020 \\
0.347-0.403\end{array}$ & $\begin{array}{c}0.417 \\
0.027 \\
0.361-0.458\end{array}$ & $\begin{array}{c}0.336 \\
0.054 \\
0.278-0.617\end{array}$ \\
\hline LF2 & $\begin{array}{l}\text { Mean } \\
\text { StdDev. } \\
\text { Range }\end{array}$ & $\begin{array}{c}0.196 \\
0.016 \\
0.167-0.222\end{array}$ & $\begin{array}{c}0.240 \\
0.023 \\
0.181-0.292\end{array}$ & $\begin{array}{c}0.312 \\
0.026 \\
0.264-0.347\end{array}$ & $\begin{array}{c}0.212 \\
0.022 \\
0.181-0.236\end{array}$ & $\begin{array}{c}0.228 \\
0.021 \\
0.194-0.278\end{array}$ & $\begin{array}{c}0.184 \\
0.024 \\
0.139-0.236\end{array}$ \\
\hline WF 1 & $\begin{array}{c}\text { Mean } \\
\text { StdDev. } \\
\text { Range }\end{array}$ & $\begin{array}{c}0.136 \\
0.011 \\
0.111-0.153\end{array}$ & $\begin{array}{c}0.147 \\
0.017 \\
0.111-0.181\end{array}$ & $\begin{array}{c}0.163 \\
0.024 \\
0.111-0.208\end{array}$ & $\begin{array}{c}0.168 \\
0.012 \\
0.153-0.181\end{array}$ & $\begin{array}{c}0.167 \\
0.018 \\
0.109-0.208\end{array}$ & $\begin{array}{c}0.125 \\
0.010 \\
0.097-0.139\end{array}$ \\
\hline WF2 & $\begin{array}{c}\text { Mean } \\
\text { StdDev. } \\
\text { Range }\end{array}$ & $\begin{array}{c}0.115 \\
0.014 \\
0.097-0.139\end{array}$ & $\begin{array}{c}0.136 \\
0.016 \\
0.097-0.167\end{array}$ & $\begin{array}{c}0.152 \\
0.022 \\
0.111-0.194\end{array}$ & $\begin{array}{c}0.149 \\
0.010 \\
0.139-0.167\end{array}$ & $\begin{array}{c}0.148 \\
0.014 \\
0.125-0.181\end{array}$ & $\begin{array}{c}0.111 \\
0.010 \\
0.097-0.125\end{array}$ \\
\hline LC & $\begin{array}{l}\text { Mean } \\
\text { StdDev. } \\
\text { Range }\end{array}$ & $\begin{array}{c}0.474 \\
0.030 \\
0.431-0.542\end{array}$ & $\begin{array}{c}0.547 \\
0.035 \\
0.43 \mathrm{I}-0.625\end{array}$ & $\begin{array}{c}0.538 \\
0.042 \\
0.444-0.597\end{array}$ & $\begin{array}{c}0.583 \\
0.031 \\
0.542-0.625\end{array}$ & $\begin{array}{c}0.574 \\
0.042 \\
0.486-0.667\end{array}$ & $\begin{array}{c}0.493 \\
0.031 \\
0.431-0.556\end{array}$ \\
\hline WC & $\begin{array}{l}\text { Mean } \\
\text { StdDev. } \\
\text { Range }\end{array}$ & $\begin{array}{c}0.216 \\
0.012 \\
0.194-0.236\end{array}$ & $\begin{array}{c}0.259 \\
0.025 \\
0.150-0.306\end{array}$ & $\begin{array}{c}0.242 \\
0.030 \\
0.153-0.278\end{array}$ & $\begin{array}{c}0.292 \\
0.013 \\
0.278-0.319\end{array}$ & $\begin{array}{c}0.274 \\
0.018 \\
0.250-0.306\end{array}$ & $\begin{array}{c}0.220 \\
0.020 \\
0.139-0.264\end{array}$ \\
\hline LP & $\begin{array}{l}\text { Mean } \\
\text { StdDev. } \\
\text { Range }\end{array}$ & $\begin{array}{c}1.623 \\
0.106 \\
1.471-1.866\end{array}$ & $\begin{array}{c}1.914 \\
0.145 \\
1.602-2.195\end{array}$ & $\begin{array}{c}1.958 \\
0.162 \\
1.574-2.265\end{array}$ & $\begin{array}{c}1.918 \\
0.143 \\
1.646-2.129\end{array}$ & $\begin{array}{c}1.991 \\
0.166 \\
1.580-2.292\end{array}$ & $\begin{array}{c}1.509 \\
0.138 \\
1.185-1.778\end{array}$ \\
\hline WP & $\begin{array}{l}\text { Mean } \\
\text { StdDev. } \\
\text { Range }\end{array}$ & $\begin{array}{c}2.026 \\
0.177 \\
1.778-2.513\end{array}$ & $\begin{array}{c}2.135 \\
0.202 \\
1.756-2.569\end{array}$ & $\begin{array}{c}2.370 \\
0.232 \\
1.795-2.762\end{array}$ & $\begin{array}{c}2.151 \\
0.185 \\
1.866-2.403\end{array}$ & $\begin{array}{c}2.649 \\
0.240 \\
2.173-3.042\end{array}$ & $\begin{array}{c}1.798 \\
0.181 \\
1.405-2.151\end{array}$ \\
\hline WPB & $\begin{array}{c}\text { Mean } \\
\text { StdDev. } \\
\text { Range }\end{array}$ & $\begin{array}{c}1.800 \\
0.173 \\
1.537-2.237\end{array}$ & $\begin{array}{c}2.042 \\
0.243 \\
1.602-2.596\end{array}$ & $\begin{array}{c}2.219 \\
0.271 \\
1.630-2.734\end{array}$ & $\begin{array}{c}1.945 \\
0.207 \\
1.668-2.348\end{array}$ & $\begin{array}{c}2.514 \\
0.264 \\
2.072-2.972\end{array}$ & $\begin{array}{c}1.732 \\
0.207 \\
1.383-2.129\end{array}$ \\
\hline WPA & $\begin{array}{l}\text { Mean } \\
\text { StdDev. } \\
\text { Range }\end{array}$ & $\begin{array}{c}1.485 \\
0.110 \\
1.317-1.712\end{array}$ & $\begin{array}{c}1.634 \\
0.147 \\
1.317-1.93\end{array}$ & $\begin{array}{c}1.796 \\
0.165 \\
1.409-2.072\end{array}$ & $\begin{array}{c}1.669 \\
0.115 \\
1.471-1.851\end{array}$ & $\begin{array}{c}2.003 \\
0.167 \\
1.685-2.273\end{array}$ & $\begin{array}{c}1.465 \\
0.133 \\
1.207-1.668\end{array}$ \\
\hline WE & $\begin{array}{c}\text { Mean } \\
\text { StdDev. } \\
\text { Range }\end{array}$ & $\begin{array}{c}2.795 \\
0.405 \\
2.292-3.948\end{array}$ & $\begin{array}{c}2.944 \\
0.376 \\
2.320-3.640\end{array}$ & $\begin{array}{c}3.168 \\
0.401 \\
2.486-3.991\end{array}$ & $\begin{array}{c}2.972 \\
0.280 \\
2.541-3.427\end{array}$ & $\begin{array}{c}3.802 \\
0.453 \\
3.007-4.633\end{array}$ & $\begin{array}{c}2.757 \\
0.381 \\
2.098-3.462\end{array}$ \\
\hline LE & $\begin{array}{l}\text { Mean } \\
\text { StdDev. } \\
\text { Range }\end{array}$ & $\begin{array}{c}4.479 \\
0.580 \\
3.728-5.942\end{array}$ & $\begin{array}{c}5.284 \\
0.658 \\
4.254-6.739\end{array}$ & $\begin{array}{c}5.323 \\
0.680 \\
4.079-6.666\end{array}$ & $\begin{array}{c}4.997 \\
0.536 \\
4.298-6.087\end{array}$ & $\begin{array}{c}6.295 \\
0.661 \\
5.123-7.392\end{array}$ & $\begin{array}{c}4.657 \\
0.656 \\
3.509-5.870\end{array}$ \\
\hline LPF & $\begin{array}{l}\text { Mean } \\
\text { StdDev. } \\
\text { Range }\end{array}$ & $\begin{array}{c}1.975 \\
0.121 \\
1.822-2.348\end{array}$ & $\begin{array}{c}1.966 \\
0.142 \\
1.712-2.458\end{array}$ & $\begin{array}{c}2.208 \\
0.211 \\
1.795-2.569\end{array}$ & $\begin{array}{c}2.142 \\
0.145 \\
1.888-2.320\end{array}$ & $\begin{array}{c}2.459 \\
0.184 \\
2.129-2.797\end{array}$ & $\begin{array}{c}1.763 \\
0.167 \\
1.383-2.085\end{array}$ \\
\hline WPF & $\begin{array}{l}\text { Mean } \\
\text { StdDev. } \\
\text { Range }\end{array}$ & $\begin{array}{c}0.482 \\
0.034 \\
0.417-0.569\end{array}$ & $\begin{array}{c}0.519 \\
0.052 \\
0.258-0.611\end{array}$ & $\begin{array}{c}0.596 \\
0.046 \\
0.500-0.667\end{array}$ & $\begin{array}{c}0.634 \\
0.074 \\
0.477-0.708\end{array}$ & $\begin{array}{c}0.660 \\
0.071 \\
0.542-0.965\end{array}$ & $\begin{array}{c}0.472 \\
0.036 \\
0.389-0.583\end{array}$ \\
\hline
\end{tabular}


TABLE 3 (continued).

\begin{tabular}{|c|c|c|c|c|c|c|c|}
\hline Character & & A. anatolicus & A. cottyi & A. gonzalezi & A. larbii & A. thalassinus & A. westringii \\
\hline LPT & $\begin{array}{l}\text { Mean } \\
\text { StdDev. } \\
\text { Range }\end{array}$ & $\begin{array}{c}2.248 \\
0.194 \\
1.932-2.623\end{array}$ & $\begin{array}{c}2.223 \\
0.160 \\
1.756-2.541\end{array}$ & $\begin{array}{c}2.446 \\
0.166 \\
2.044-2.734\end{array}$ & $\begin{array}{c}2.196 \\
0.129 \\
1.976-2.320\end{array}$ & $\begin{array}{c}2.661 \\
0.187 \\
2.265-2.972\end{array}$ & $\begin{array}{c}1.841 \\
0.150 \\
1.558-2.173\end{array}$ \\
\hline WPT & $\begin{array}{c}\text { Mean } \\
\text { StdDev. } \\
\text { Range }\end{array}$ & $\begin{array}{c}0.339 \\
0.023 \\
0.306-0.403\end{array}$ & $\begin{array}{c}0.390 \\
0.038 \\
0.319-0.472\end{array}$ & $\begin{array}{c}0.412 \\
0.044 \\
0.319-0.500\end{array}$ & $\begin{array}{c}0.448 \\
0.042 \\
0.389-0.528\end{array}$ & $\begin{array}{c}0.468 \\
0.040 \\
0.389-0.569\end{array}$ & $\begin{array}{c}0.337 \\
0.031 \\
0.264-0.417\end{array}$ \\
\hline LT1 & $\begin{array}{l}\text { Mean } \\
\text { StdDev. } \\
\text { Range }\end{array}$ & $\begin{array}{c}0.510 \\
0.042 \\
0.417-0.611\end{array}$ & $\begin{array}{c}0.451 \\
0.041 \\
0.361-0.583\end{array}$ & $\begin{array}{c}0.547 \\
0.058 \\
0.486-0.695\end{array}$ & $\begin{array}{c}0.477 \\
0.043 \\
0.417-0.528\end{array}$ & $\begin{array}{c}0.595 \\
0.042 \\
0.542-0.695\end{array}$ & $\begin{array}{c}0.447 \\
0.038 \\
0.375-0.556\end{array}$ \\
\hline LT2 & $\begin{array}{l}\text { Mean } \\
\text { StdDev. } \\
\text { Range }\end{array}$ & $\begin{array}{c}0.384 \\
0.034 \\
0.306-0.444\end{array}$ & $\begin{array}{c}0.364 \\
0.030 \\
0.306-0.444\end{array}$ & $\begin{array}{c}0.357 \\
0.041 \\
0.292-0.431\end{array}$ & $\begin{array}{c}0.340 \\
0.022 \\
0.306-0.375\end{array}$ & $\begin{array}{c}0.420 \\
0.048 \\
0.319-0.514\end{array}$ & $\begin{array}{c}0.302 \\
0.027 \\
0.250-0.361\end{array}$ \\
\hline LT3 & $\begin{array}{c}\text { Mean } \\
\text { StdDev. } \\
\text { Range }\end{array}$ & $\begin{array}{c}0.292 \\
0.025 \\
0.250-0.347\end{array}$ & $\begin{array}{c}0.317 \\
0.028 \\
0.250-0.375\end{array}$ & $\begin{array}{c}0.315 \\
0.042 \\
0.222-0.375\end{array}$ & $\begin{array}{c}0.328 \\
0.023 \\
0.292-0.361\end{array}$ & $\begin{array}{c}0.372 \\
0.040 \\
0.297-0.472\end{array}$ & $\begin{array}{c}0.247 \\
0.024 \\
0.208-0.319\end{array}$ \\
\hline LON & $\begin{array}{l}\text { Mean } \\
\text { StdDev. } \\
\text { Range }\end{array}$ & $\begin{array}{c}0.563 \\
0.036 \\
0.486-0.639\end{array}$ & $\begin{array}{c}0.557 \\
0.047 \\
0.444-0.681\end{array}$ & $\begin{array}{c}0.609 \\
0.046 \\
0.528-0.722\end{array}$ & $\begin{array}{c}0.601 \\
0.028 \\
0.556-0.639\end{array}$ & $\begin{array}{c}0.651 \\
0.035 \\
0.569-0.708\end{array}$ & $\begin{array}{c}0.544 \\
0.039 \\
0.486-0.625\end{array}$ \\
\hline WT1 & $\begin{array}{l}\text { Mcan } \\
\text { StdDev. } \\
\text { Range }\end{array}$ & $\begin{array}{c}0.280 \\
0.019 \\
0.250-0.333\end{array}$ & $\begin{array}{c}0.322 \\
0.033 \\
0.250-0.403\end{array}$ & $\begin{array}{c}0.310 \\
0.035 \\
0.250-0.375\end{array}$ & $\begin{array}{c}0.323 \\
0.031 \\
0.292-0.375\end{array}$ & $\begin{array}{c}0.361 \\
0.028 \\
0.296-0.417\end{array}$ & $\begin{array}{c}0.235 \\
0.017 \\
0.208-0.278\end{array}$ \\
\hline WT2 & $\begin{array}{l}\text { Mean } \\
\text { StdDev. } \\
\text { Range }\end{array}$ & $\begin{array}{c}0.263 \\
0.018 \\
0.236-0.306\end{array}$ & $\begin{array}{c}0.305 \\
0.034 \\
0.222-0.397\end{array}$ & $\begin{array}{c}0.295 \\
0.039 \\
0.236-0.375\end{array}$ & $\begin{array}{c}0.307 \\
0.032 \\
0.250-0.347\end{array}$ & $\begin{array}{c}0.352 \\
0.030 \\
0.278-0.403\end{array}$ & $\begin{array}{c}0.217 \\
0.017 \\
0.181-0.264\end{array}$ \\
\hline WT3 & $\begin{array}{l}\text { Mean } \\
\text { StdDev. } \\
\text { Range }\end{array}$ & $\begin{array}{c}0.391 \\
0.032 \\
0.347-0.472\end{array}$ & $\begin{array}{c}0.460 \\
0.041 \\
0.361-0.569\end{array}$ & $\begin{array}{c}0.453 \\
0.052 \\
0.361-0.569\end{array}$ & $\begin{array}{c}0.467 \\
0.026 \\
0.428-0.514\end{array}$ & $\begin{array}{c}0.524 \\
0.046 \\
0.417-0.625\end{array}$ & $\begin{array}{c}0.354 \\
0.026 \\
0.292-0.417\end{array}$ \\
\hline WON & $\begin{array}{l}\text { Mean } \\
\text { StdDev. } \\
\text { Range }\end{array}$ & $\begin{array}{c}0.139 \\
0.011 \\
0.125-0.167\end{array}$ & $\begin{array}{c}0.159 \\
0.013 \\
0.139-0.181\end{array}$ & $\begin{array}{c}0.160 \\
0.015 \\
0.139-0.181\end{array}$ & $\begin{array}{c}0.160 \\
0.010 \\
0.139-0.167\end{array}$ & $\begin{array}{c}0.190 \\
0.010 \\
0.181-0.208\end{array}$ & $\begin{array}{c}0.138 \\
0.011 \\
0.125-0.167\end{array}$ \\
\hline
\end{tabular}

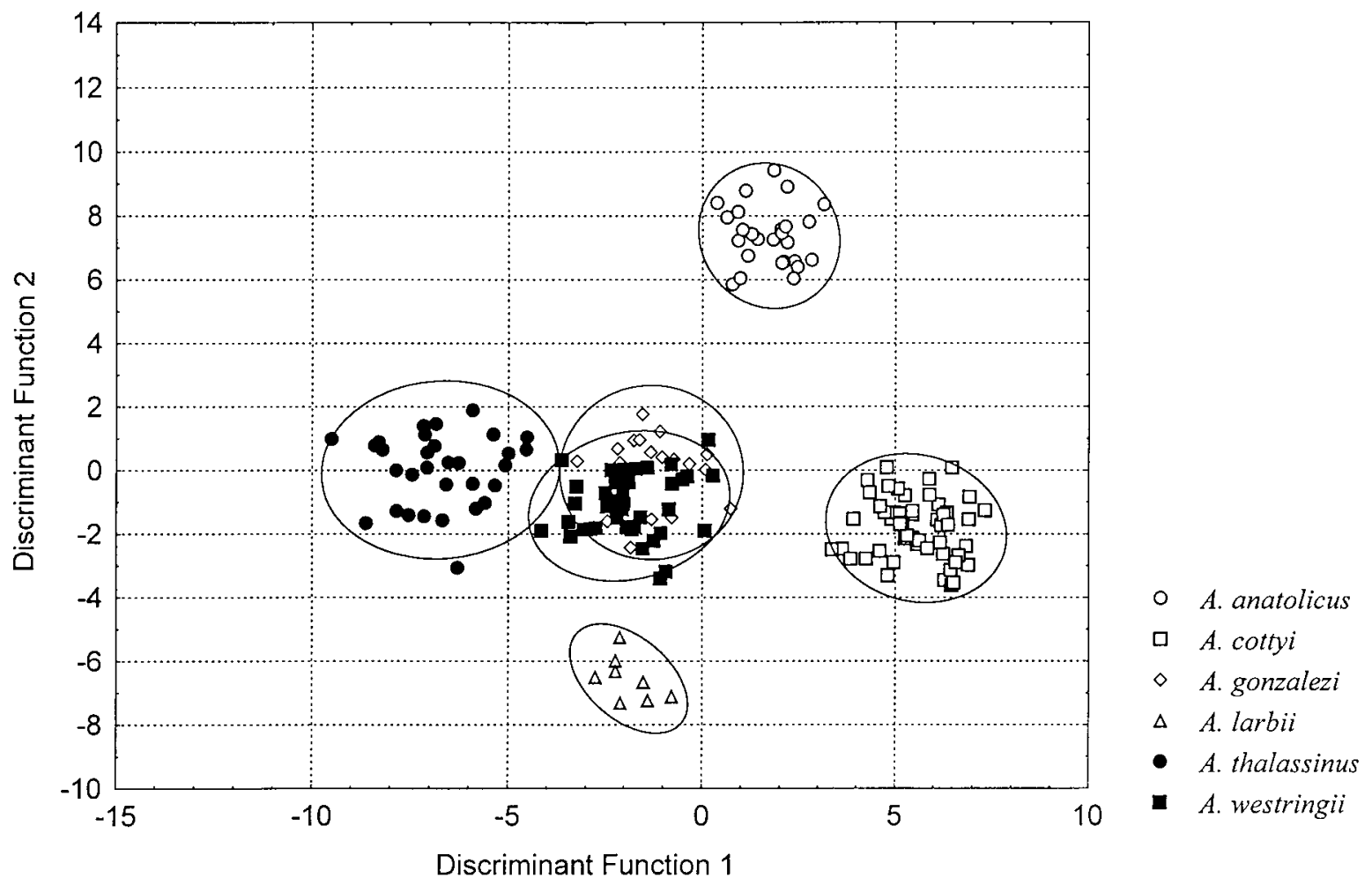

Fig. 1. Plot of all Aspidiotes specimens onto the first and second discriminant functions based on a set of 30 morphometric characters. Circles include $95 \%$ of specimens in each species. 


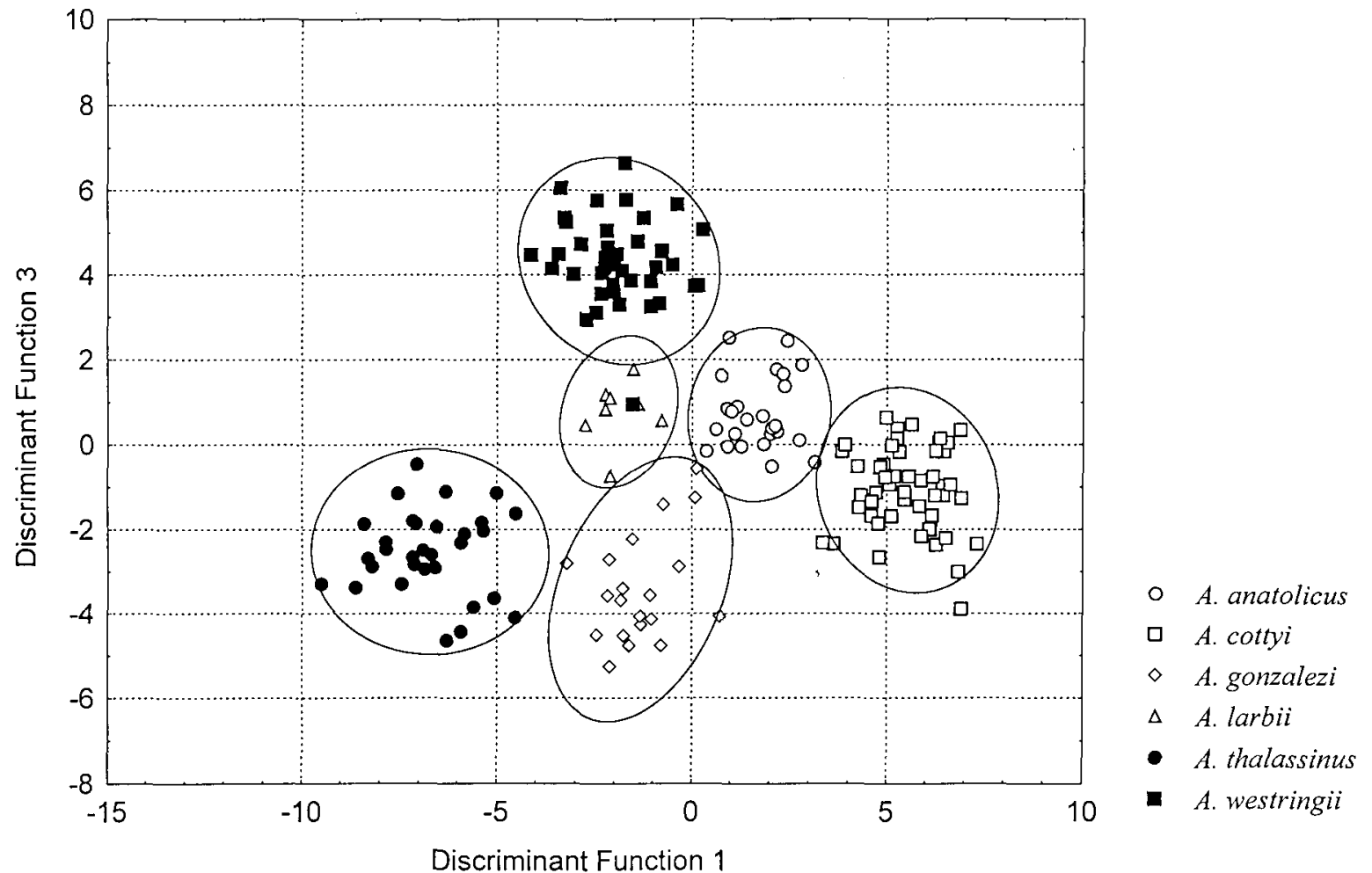

Fig. 2. Plot of all Aspidiotes specimens onto the first and third discriminant functions based on a set of 30 morphometric characters. Circles include $95 \%$ of the specimens in each species.

Individual specimens are projected onto the first two discriminant functions in Fig. 1, and onto the first and third functions in Fig. 2. Because all species were clearly separated in the discriminant space defined by the first three functions, the fourth function was not used.

The first discriminant function explains $44 \%$ of total variation (Table 4), and mainly separates $A$. thalassinus from $A$. cottyi. The other four species, clustered in the middle, cannot be discriminated by this function. From the standardized coefficients (Table 4), the characters that have the greatest weight on this function (characters most discriminatory) are the lengths of scape (LSC) and pronotum (LP) and, to a lesser extent, the elytral length (LE). LSC is opposite in sign to LP and LE. In general $A$. thalassimus is larger than $A$. cottyi. The length of the pronotum (LP), however, is very similar in both species: average $1.99 \mathrm{~mm}$ in $A$. thalassinus and $1.91 \mathrm{~mm}$ in $A$. cottyi (Table 3). Likewise, the differences between the elytral length (LE) in both species $(5.284-6.295 \mathrm{~mm})$ do not correspond to the general differences in size. The scape, however, is much longer in $A$. thalassinus (1.174) than in A. cottyi $(0.734)$. That is $A$. thalassimus is characterized by a proportionally shorter pronotum, shorter elytra, and a very long antennal scape, whereas $A$. cotty $i$ has a longer pronotum, elongated elytra, and a shorter scape.

The second discriminant function accounts for $27 \%$ of total variation. A. larbii and A. anatolicus are clearly discriminated by this function while the other four species are clustered in the middle (Fig. 1). The contrast between the length of rostrum (LR) and length of clytra (LE) is responsible for this discrimination. A. larbit is larger than A. anatolicus, but in proportion $A$. anatolicus has a longer
TABLI: 4. Standardized coefficients of the first four discriminant functions separating the six species of Aspidiotes. In bold, characters with the greatest weight in the function.

\begin{tabular}{|c|c|c|c|c|}
\hline Character & DF 1 & $\mathrm{DF} 2$ & DF 3 & DF4 \\
\hline WRP & -0.451 & -0.568 & -0.036 & -0.160 \\
\hline WRS & -0.644 & -0.600 & -0.258 & -0.372 \\
\hline LR & -0.341 & 1.169 & 0.940 & -0.168 \\
\hline WF & 0.031 & 0.743 & -0.591 & -0.289 \\
\hline LSC & -1.179 & -0.638 & -0.024 & 0.252 \\
\hline WSC & 0.169 & -0.281 & 0.149 & 0.352 \\
\hline $\mathrm{LFI}$ & 0.038 & -0.101 & 0.036 & 0.168 \\
\hline $\mathrm{LF} 2$ & 0.281 & 0.046 & 0.374 & 0.632 \\
\hline WF 1 & 0.025 & 0.127 & -0.244 & -0.136 \\
\hline$W F 2$ & -0.141 & -0.312 & 0.082 & 0.152 \\
\hline $\mathrm{LC}$ & 0.429 & -0.316 & 0.048 & -0.124 \\
\hline WC & -0.002 & -0.350 & -0.138 & -0.153 \\
\hline LP & 1.489 & -0.787 & 0.227 & 0.442 \\
\hline WP & -0.047 & 0.511 & 0.219 & 0.407 \\
\hline WPB & -0.639 & 0.136 & -0.187 & -0.705 \\
\hline WPA & 0.103 & -0.192 & 0.433 & 0.254 \\
\hline WI: & 0.076 & 0.731 & 0.067 & 0.577 \\
\hline I.F & 0.860 & -1.428 & -0.274 & -0.965 \\
\hline $\mathrm{L}, \mathrm{Pl}$ & 0.057 & 0.443 & 0.022 & 0.032 \\
\hline WPF & -0.103 & -0.037 & 0.123 & 0.198 \\
\hline LPT & 0.068 & 0.492 & 0.501 & 0.068 \\
\hline WPT & 0.126 & -0.246 & -0.432 & 0.437 \\
\hline LTI & -0.300 & 0.380 & -0.222 & 0.258 \\
\hline $\mathrm{LT} 2$ & 0.128 & 0.571 & -0.234 & -0.360 \\
\hline LT3 & -0.003 & 0.102 & 0.211 & -0.440 \\
\hline LON & -0.180 & 0.189 & -0.734 & 0.510 \\
\hline WT 1 & 0.428 & -0.120 & -0.320 & -0.352 \\
\hline WT2 & -0.179 & 0.035 & 0.575 & -0.027 \\
\hline WT3 & -0.171 & -0.178 & 0.328 & -0.320 \\
\hline WON & -0.031 & -0.118 & 0.085 & -0.461 \\
\hline $\begin{array}{l}\text { Percentage of } \\
\text { explaincd variance }\end{array}$ & $44 \%$ & $27 \%$ & $17 \%$ & $9 \%$ \\
\hline Eigenvalue & 19.01 & 11.55 & 7.47 & 3.73 \\
\hline $\begin{array}{l}\text { Cumulative } \\
\text { variance }\end{array}$ & 0.44 & 0.71 & 0.88 & 0.97 \\
\hline
\end{tabular}




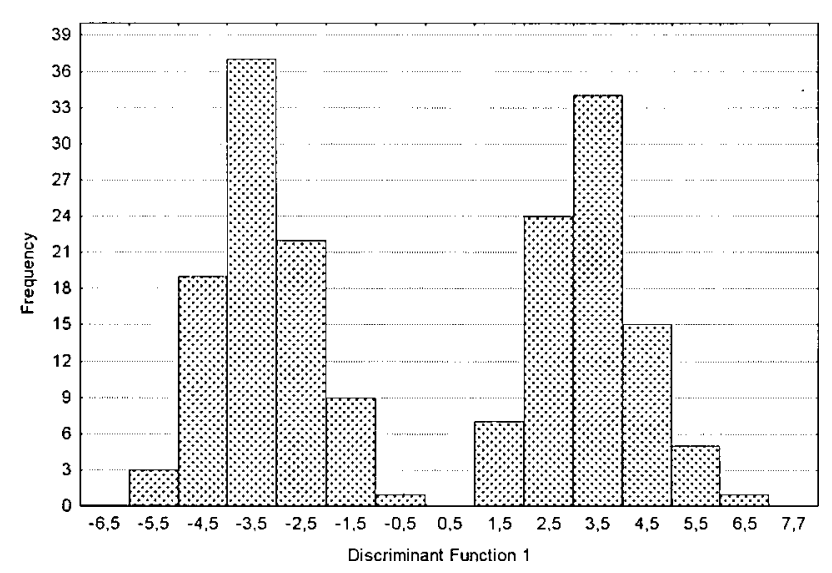

Fig. 3. Frequency distribution of Aspidiotes specimens along the first discriminant function. Left - females; right - males.

rostrum and shorter elytra than $A$. larbii. This shape difference is reinforced by contrasts in three other variables: frons width between the eyes (WF) and elytral width (WE) against length of pronotum (LP).

As seen in Fig. 1, the discriminant space defined by the first two functions allows us to clearly separate four species of the genus: $A$. thalassinus, A. cottyi, A. anatolicus, and $A$. larbii.

TABLE 5. Standardized coefficients of the Fisher's linear discriminant function separating specimens of Aspidiotes by sex. In bold, characters with the greatest weight in the function.

\begin{tabular}{lr}
\hline Character & DF1 \\
\hline WRP & $\mathbf{1 . 1 2 6}$ \\
WRS & 0.481 \\
LR & -0.014 \\
WF & -0.145 \\
LSC & 0.561 \\
WSC & -0.011 \\
LF1 & -0.101 \\
LF2 & -0.306 \\
WF1 & -0.207 \\
WF2 & 0.808 \\
LC & 0.101 \\
WCM & 0.224 \\
LP & -0.376 \\
WP & 0.713 \\
WPB & -1.186 \\
WPA & -0.327 \\
WE & -1.751 \\
LE & -1.574 \\
LPF & -0.304 \\
WPF & 0.090 \\
LPT & -0.145 \\
WPT & -0.072 \\
LT1 & 0.371 \\
LT2 & 0.653 \\
LT3 & 0.175 \\
LON & -0.066 \\
WT1 & 0.564 \\
WT2 & 0.046 \\
WT3 & 0.683 \\
WON & 0.341 \\
Percentage of variance explained & $100 \%$ \\
Eigenvalue & 11.30 \\
Cumulative variance & 1.00 \\
\hline
\end{tabular}

The third discriminant function explains $17 \%$ of total variation. This function morphologically separates the two remaining species (Fig. 2): A. gonzalezi from $A$. westringii, by an increase in the length of rostrum (LR) and decrease in the length of onychium (LON).

The DFA classification functions, based on linear combinations of the original variables, correctly identified all specimens, thus demonstrating the efficacy of this set of morphometric characters for identifying species of $\mathrm{As}$ pidiotes.

(ii) (b) MANOVA revealed highly significant differences (Wilks' lambda $30.191=0.081765 ; p<0.001$ ) between males and females within the genus Aspidiotes. Subsequent MANOVAs revealed sexual dimorphism in five species: $A$. thalassimus, $A$. cottyi, $A$. anatolicus, $A$. westringii and $A$. gonzalezi $(p<0.001)$. A discriminant analysis, to separate specimens by sex, provided one discriminant function, allowing for complete discrimination of males and females within the genus (Fig. 3). Based on the standardized coefficients, the weight of this function is mainly dependent on: width of pronotum at base (WPB), width of elytra (WE), length of elytra (LE), and width of rostrum on pterigia (WRP) (Table 5). Females have wider, longer elytra and a wider pronotum than males whereas males have a wider rostrum.

Table 6 shows classification functions that separate specimens by sex and the percentage of correct attributions $(100 \%)$. These classification functions can serve as an additional diagnostic tool for determining the sex of specimens. This may substitute the study of genitalia, which has been often necessary in order to distinguish males from females in Aspidiotes.

(iii) The dendrogram (Fig. 4) constructed with UPGMA based on Mahalanobis' distance values, shows the morphometric relationships between the six species of the genus. This dendrogram shows the same patterns of association as found previously in the plots of the first three discriminant functions. At the base of the dendrogram there is the first separation, which separates $A$. anatolicus and $A$. cottyi from the other four species; within the latter there are two subclusters: $A$. gonzalezi-A. thalassinus and $A$. larbii-A.westringii. It should be noted

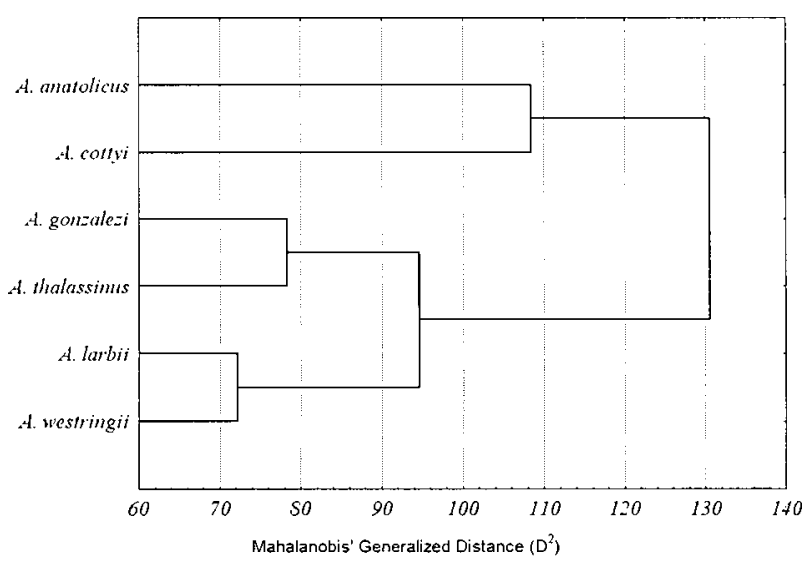

Fig. 4. UPGMA dendrogram of Mahalanobis' generalized distances showing the morphometric relationships between the species of Aspidiotes. 


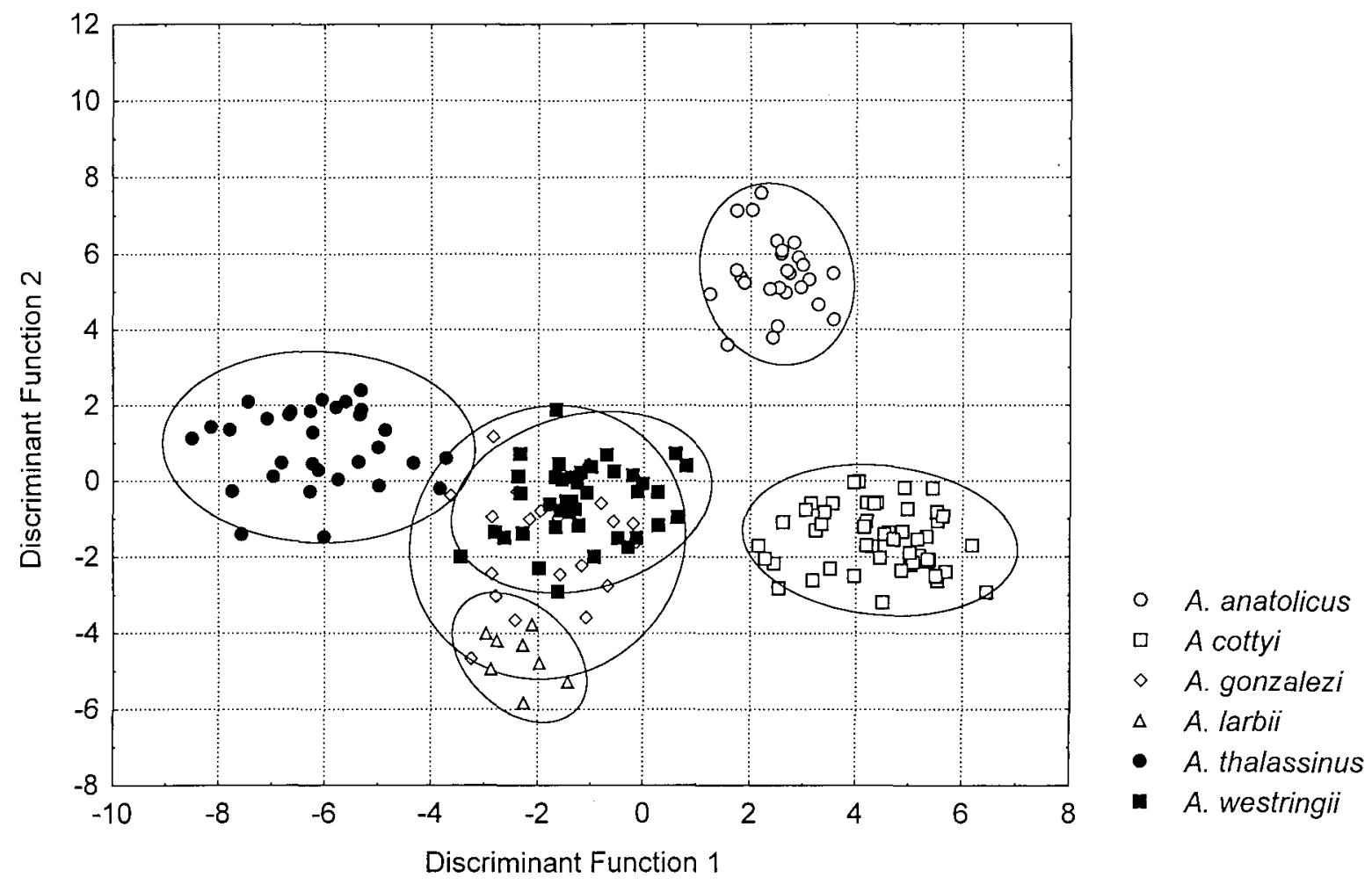

Fig. 5. Plot of all Aspidiotes specimens onto the first and second discriminant functions based on a reduced set of 15 morphometric characters (see text).

TABßLE 6. Classification functions used to determine the sex of specimens of Aspidiotes and the percentage of correct attributions.

\begin{tabular}{lrr}
\hline Character & remalcs & Males \\
\hline WRP & -98.926 & -45.082 \\
WRS & 38.139 & 70.357 \\
LR & -21.259 & -21.709 \\
WF & 52.455 & 45.455 \\
LSC & -111.112 & -90.813 \\
WSC & 65.916 & 63.205 \\
LF1 & 33.175 & 22.047 \\
LF2 & 30.260 & -15.716 \\
WF1 & -221.049 & -284.883 \\
WF2 & -9.307 & 257.394 \\
LC & 224.056 & 237.767 \\
WC & 0.256 & 0.304 \\
LP & 39.486 & 28.799 \\
WP & -35.206 & -20.911 \\
WPB & -28.523 & -53.362 \\
WPA & 99.006 & 89.232 \\
WE & 25.330 & -0.660 \\
LE & -4.935 & -19.474 \\
LPF & -11.546 & -18.736 \\
WPF & -9.285 & -2.383 \\
LPT & 33.674 & 30.613 \\
WPT & 12.988 & 4.899 \\
LT1 & 98.715 & 133.364 \\
LT2 & 7.074 & 92.942 \\
LT3 & -270.805 & -247.107 \\
LON & 289.669 & 281.752 \\
WT1 & -191.796 & -117.017 \\
WT2 & -153.107 & -147.196 \\
WT3 & -44.464 & 22.541 \\
WON & 298.142 & 403.545 \\
\hline Constant & -156.870 & -156.691 \\
\hline \%of correctly classificd & $100 \%$ & $100 \%$ \\
\hline & &
\end{tabular}

that a considerable distance $\left(D^{2}=130\right)$ separates the first two clusters, indicating that $A$. anatolicus and $A$. cotty $i$ are very different morphometrically from the other species, and also very different from each other $\left(D^{2}=108\right)$. Even within the second cluster the four species are significantly separated ( $\mathrm{D}^{2}$ ranges from 72 to 78 ). In short, there are marked morphometric differences between the species.

\section{Statistical reliability of our results}

Because the specimen to variable ratio is relatively low $(\cong 6)$, our results may be statistically unreliable. Typically, a minimum ratio of $10-20$ is considered necessary for reliability. The number of specimens was limited and any a priori selection among the 30 characters, obtained after a preliminary study, was not feasible, as all of them differed significantly between species $(p<0.00001)$.

As a check of the accuracy of our results, we performed a multivariate analysis using a reduced set of 15 characters. This included those characters that proved the best discriminators among species in the previous analysis; we also excluded those characters that were redundant (as judged by the tolerance values), and summed others into a single character (e.g., LT1 + LT2 + LT3). In addition, results from this analysis (having greater reliability but poorer resolution), if consistent with the first 30 characters study, would provide strong support for our conclusions. The results of this analysis conformed with those of the previous analysis. Although there was a loss of resolution (i.e., morphometric differences between species were lower), both the characters and the species discriminated were the same (Fig. 5). We believe that this 


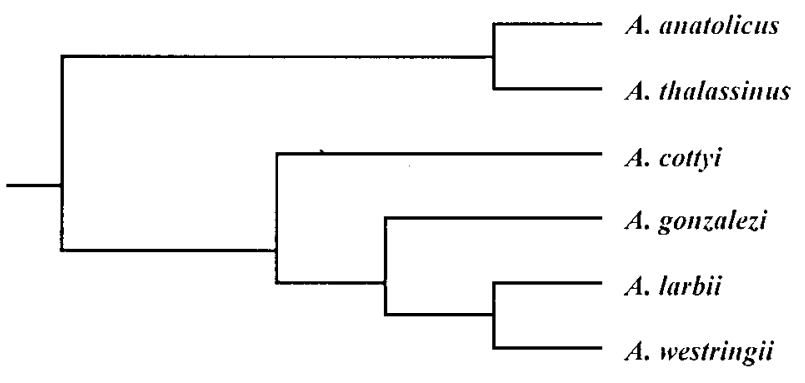

Fig. 6. Cladogram depicting the phylogenetic relationships between the species of Aspidiotes as proposed by Sánchez-Ruiz \& Alonso-Zarazaga (1994).

two-step procedure gets over the problem of limited numbers of specimens.

\section{DISCUSSION}

\section{Character efficacy}

On the basis of this study it is evident that morphometric characters can be used to separate species within the genus Aspidiotes. The set of morphometric characters herein analysed has proved effective in species discrimination, in particular, lengths of rostrum, scape, onychium and pronotum, and width and length of elytra.

Despite differences in their average values, the ranges in morphometric characters overlapped to some extent between species (Table 3). No character alone can be used for full discrimination. That is, species can only be separated on the basis of all the characters. Thus, taxonomic discrimination requires a multivariate approach: species overlap when characters are used individually but become distinct entities when many characters are considered jointly (Foottit \& Sorensen, 1992). This is a consequence of the intercorrelation of characters, itself derived from the epistatic or pleiotropic interactions among genes coding them (Leamy \& Sustarsic, 1978; Atchley et al., 1982).

The genus Aspidiotes shows clear sexual morphometric dimorphism. Differences between sexes occur in the width of rostrum on pterigia, width of pronotum at base, and width and length of elytra. The last three characters differ in size between males and females, as in other genera of Curculionidae. Females are generally larger than males. This size difference, however, is more difficult to appreciate in Aspidiotes without the help of multivariate analysis.

In addition, the classification functions provided by discriminant analysis can be used as a diagnostic tool when discrete characters are unreliable. They have the advantage of identifying "problematic" specimens objectively, and their efficacy can be evaluated by the percentage of correct identifications.

\section{Systematic implications}

The comparison of the dendrogram in Fig. 4, portraying the morphometric relationships between species, with the cladogram in Fig. 6, representing their phylogenetic relationships (Sánchez-Ruiz \& Alonso-Zarazaga, 1994), show notable differences. The species closely clustered in the dendrogram, such as $A$. anatolicus- $A$. cottyi or $A$. thalassinus- $A$. gonzalezi, are very far apart in the cladogram, and are even included in different subgenera, Phaenognathus and Aspidiotes s. str., respectively (Sánchez-Ruiz \& Alonso-Zarazaga, 1994). Conversely, the species that are closely related in the cladogram and belong to the same subgenus ( $A$. anatolicus- $A$. thalassimis) showed the greatest morphometric differences in the phenogram, even greater than in species from different subgenera. The only exception occurs in the pair $A$. larbii-A. westringii, which appear closely related in both figures.

This tends to contradict the claim of Sorensen \& Foottit (1992) about the potential use of discriminant function analysis in phylogenetic inference. These authors claim that the DFA-based dendrogram reflects not only morphometric similarity but also the phylogenetic relationships between species. Using Lande's (1979) phenotypic model for multivariate evolution, discriminant functions would represent "historical gradients of selective pressure that the species have been exposed to during their common evolutionary history" (Sorensen \& Foottit, 1992). This approach has been criticized, however, because of the lack of biological meaning of discriminant functions (Crespi \& Bookstein, 1989; Crespi, 1992). To date, the DFA model has been used several times (Schluter, 1984; Sorensen, 1987; Wood \& Pesek, 1992; Simon, 1992). In these studies, the DFA-based dendrograms largely corroborated previous cladograms for the same groups. In our case, there was no congruence between the relationships depicted by the cladogram and the phenogram. Because phylogenetic arguments have a more sound theoretical base than phenetics, it is more likely that the cladogram reflects the phylogenetic relationships between species of Aspidiotes whereas the phenogram only depicts their morphometric relationships.

The comparison of the distributions of the species in both figures revealed other important differences. The species that are closely related in the cladogram have similar geographic distributions. This suggests that the species could have originated from a succesion of vicariance events on a widespread ancestor, with every vicariance being followed by a speciation event (Sánchez-Ruiz \& Alonso-Zarazaga, 1994). In the phenogram, in constrast, those species occurring in the same or adjacent geographical areas are separated. This is the case for the species pairs $A$. anatolicus (Turkey)-A. thalassinus (Greece + Turkey), and A. cottyi (Morocco)-A. larbii (Morocco). In each pair, both species belong to the same subgenus, Phaenognathus or Aspidiotes, respectively (Fig. 6). The only apparent exception to this pattern is the species-pair $A$. larbii-A. westringii, which appears closely clustered both in the cladogram and in the phenogram. They have allopatric geographic distributions: $A$. westringii occurs in southeastern Spain whereas $A$. larbii is apparently restricted to the westernmost foothills of the High Atlas (Mogador) (Sánchez-Ruiz \& Alonso-Zarazaga, 1994).

Therefore, two conclusions can be drawn from a comparison of the DFA-phenogram and the cladogram of $\mathrm{As}$ pidiotes: (1) The contradictory form of the species 
relationships depicted by both methods. The most similar species based on discrete characters are in terms of morphometric characters the most different. (2) The largest morphometric differences were between closely related species of Aspidiotes (i.e., belonging to the same subgenus), which share a similar geographic distribution (sympatric species).

Morphometric changes are evolutionarily less expensive than discrete changes, which imply the modification or disappearance of a structure. The latter necessarily require disassociations or rearrangements of genetic linkages so discrete changes are more expensive from an energetic viewpoint, and evolutionarily constrained (Sorensen \& Foottit, 1992). Morphometric changes only require arrangements of a few pleiotropically correlated genes. Thus, a large morphometric divergence could simply arise from a small number of genetic divergences (Atchley et al., 1982).

For species belonging to the same subgenus, that share very similar genetic covariance matrices and have the same geographic distribution (i.e., subjected to similar selection pressures), "morphometric variance may be the last and easiest way to diverge during evolution because of lower evolutionary energy constraints" (Sorensen, 1991). Therefore, morphometric differences have proved to be more important for closely related and sympatric species of Aspidiotes whereas divergence in discrete characters is more important in the case of distantly related species (belonging to different subgenera). Thus, the phenogram in Fig. 4 mainly reflects the recent morphometric divergence between closely related, sympatric species of Aspidiotes whereas the cladogram in Fig. 6 would actually reflect the evolution of the genus.

ACKNOWLEDGEMENTS. We thank M.A. Alonso-Zarazaga and J.M. Lobo, from the Museo Nacional de Ciencias Naturales in Madrid, and M.J. Cavalcanti, from Universidade Santa Ursula in Rio de Janeiro, for their helpful suggestions for improving this manuscript. This study was supported by the Project Fauna Iberica (PB95-0235-DGICYT).

\section{REFERENCES}

Albritci G.H., Gelvin B.R. \& Hartman S.E. 1993: Ratios as a size adjustment in morphometrics. Am. J. Phys. Anthropol. 91: 441-468.

Alonso-Znrazaga M.A. \& Sánchez-Ruiz M. 1990: Datos preliminares para la revisión del género Amomphus Schoenherr, 1848 (Col.; Curculionidae, Polydrusinae). In: IV Congreso Ibérico de Entomología. (Abstracts.) Universitat Autónoma de Barcelona, p. 112.

Atchley W.R., Nordieim E.V., Gunsett F.C. \& Crump P.L. 1982: Geometric and probabilistic aspects of statistical distance functions. Syst. Zool. 31: 445-460.

ClARIDGE M.F. \& GilliIAM C. 1992: Variation in populations of leafhoppers and planthoppers (Auchenorrhyncha): Biotypes and biological species. In Sorensen R.G \& Foottit J.T. (eds): Ordination in the Study of Morphology, Evolution and Systematics of Insects. Applications and Quantitative Genetic Rationals. Elsevier, Amsterdam, pp. 241-259.

CRESPI B.J. 1992: Natural selection and morphometrics. In Sorensen R.G \& Foottit J.T. (eds): Ordination in the Study of Morphology, Evolution and Systematics of Insects. Applica- tions and Quantitative Genetic Rationals. Elsevier, Amsterdam, pp. 55-64.

CREsP B.J. \& Bookstein F.L. 1989: A path-analytic model for the measurement of selection on morphology. Evolution 43: $18-28$.

DALY H.V. 1992: A statistical and empirical evaluation of some morphometric variables of honey bee classification. In Sorensen R.G \& Foottit J.T. (eds): Ordination in the Study of Morphology, Evolution and Systematics of Insects. Applications and Quantitative Genetic Rationals. Elsevier, Amsterdam, pp. 127-153.

Foоттा R.G. 1992: The use of ordination methods to resolve problems of species discrimination in the genus Cinara Curtis (Homoptera: Aphidoidea: Lachnidae). In Sorensen R.G \& Foottit J.T. (eds): Ordination in the Study of Morphology, Evolution and Systematics of Insects. Applications and Quantitative Genetic Rationals. Elsevier, Amsterdam, pp. 193-219.

Footrit R.G. \& SORENSEN J.T. 1992: Ordination methods: their contrast to clustering and cladistic techniques. In Sorensen R.G \& Foottit J.T. (eds): Ordination in the Study of Morphology, Evolution and Systematics of Insects. Applications and Quantitative Genetic Rationals. Elsevier, Amsterdam, pp. $1-10$.

Godwin P.A., Valintine H.T. \& Odell T.M. 1982: Identification of Pissodes strobi, P. approximatus, and P. nemorensis (Coleoptera: Curculionidae), using discriminant analysis. Ann. Entomol. Soc. Am. 75: 599-604.

HoRng S.B. \& PENG W.K. 1983: Morphological comparison of pronotum and scutellum between rice weevil and maize weevil. Natn. Taiwan Univ. Phytopathol. Entomol. 10: 39-46.

Houck M.A. 1992: Morphological variation in an ectoparatsite: Partitioning ecological and evolutionary influences. In Sorensen R.G \& Foottit J.T. (eds): Ordination in the Study of Morphology, Evolution and Systematics of Insects. Applications and Quantitative Genetic Rationals. Elsevier, Amsterdam, pp. 277-308.

LANDE R. 1979: Quantitative genetic analysis of multivariate evolution applied to brain: body size allometry. Evolution 33: 402-416.

Lenmy L. \& Sustarsic S. 1978: A morphometric discriminant analysis of agouti genotypes in C57BL/6 house mice. Syst. Zool. 27: 49-60.

Oosteribroek P. \& Arntzen J.W. 1992: Area-cladograms of circum-Mediterranean taxa in relation to Mediterranean palaeogeography. J. Biogeogr. 19: 3-20.

RICF: W.R. 1989: Analyzing tables of statistical tests. Evolution 43: $223-225$.

Sinchez-Ruiz M. \& Alonso-Zaraznga M.A. 1994: Revision of the genus Aspidiotes Schoenherr, 1847 (Coleoptera: Curculionidae, Tanymecini). Entomol. Scand. 25: 275-294.

SANMARTín I. \& MArTín-Piera F. 1999: A morphometric approach to the taxonomy of the genus Ceramida (Coleoptera: Scarabaeoidea: Melolonthidae). Can. Entomol. 131: 573-592.

Schiluter D. 1984: Morphological and phylogenetic relationships among the Darwin's Finches. Evolution 38: 921-930.

SIMON C. 1992: Discriminant analysis of year classes of periodical cicada based on wing morphometric data enhanced by molecular information. In Sorensen R.G \& Foottit J.T. (eds): Ordination in the Study of Morphology, Evolution and Systematics of Insects. Applications and Quantitative Genetic Rationals. Elsevier, Amsterdam, pp. 309-324.

Sitts R.W. \& Willig M.R. 1994: Efficacy of mensural characters in discriminating among species of Naucoridae (Insecta: Hemiptera): Multivariate approaches and ontogenetic perspectives. Ann. Entomol. Soc. Am. 87: 803-814. 
Sneatil P.H.A. \& Sokal R.R. 1973: Numerical Taxonomy: The Principles and Practice of Numerical Classification. Freeman, San Francisco, 573 pp.

SORENSEN J.T. 1987: Multivariate statistical approach to deduction of phylogeny within Essigiella [sic] (Aphididae: Lachninae). In Holman J., Pelikán J., Dixon A.F.G. \& Weismann L. (eds): Population, Structure, Genetics and Taxonomy of Aphids and Thysanoptera. Proceedings of International Symposia, Held at Smolenice, Czechoslovakia, September 9-14, 1985. SPB Academic Publishing, The Hague, pp. 243-260.

SORENSEN J.T. 1991: Phylogenetic character responses for shape component variance during the multivariate evolution of eulachnine aphids: Redescription of Pseudessigella Hille Ris Lambers (Homoptera: Aphididae: Lachninae). Pan-Pac. Entomol. 67: 28-54.

SOREnSEn J.T. \& Foottit R.G. 1992: The evolutionary quantitative genetic rationals for the use of ordination analysis in sys- tematics: Phylogenetic implications. In Sorensen R.G \& Foottit J.T. (eds): Ordination in the Study of Morphology, Evolution and Systematics of Insects. Applications and Quantitative Genetic Rationals. Elsevier, Amsterdam, pp. 29-53.

STATSOFT INC, 1996: STATISTICA for Windows (computer program manual). Tulsa, OK.

Tizndo E.J. \& Nieto-NAFrí J.M. 1994: A new species of Uroleucon (Hom. Aphididae) on Andryala spp.: A multivariate analysis. Can. Entomol. 126: 1251-1261.

WOOD T.K. \& PESEK J.D. 1992: A source of confusion or panacea in systematic studies of treehoppers (Homoptera: Membracidae)? In Sorensen R.G \& Foottit J.T. (eds): Ordination in the Study of Morphology, Evolution and Systematics of Insects. Applications and Quantitative Genetic Rationals. Elsevier, Amsterdam, pp. 349-384.

Received September 14, 1998; accepted March 4, 1999 